\title{
Adenomas hipofisarios no funcionantes: epidemiología, clínica y evolución posquirúrgica
}

\author{
Carlos Pérez-López, Alexis J. Palpán, María J. Abenza-Abildúa, Álvaro Zamarrón, Carolina Alfonso, \\ Cristina Álvarez-Escolá, Beatriz Lecumberri, Alberto Isla
}

Introducción. Los adenomas hipofisarios no funcionantes son el grupo tumoral más frecuente en la región selar. Suelen
ser neoplasias benignas diagnosticadas por síntomas visuales u hormonales, aunque no es infrecuente detectarlos como
un hallazgo casual. Objetivo. Analizar los aspectos clínicos hallados en esta enfermedad y su respuesta tras el tratamiento quirúrgico.

Pacientes y métodos. En una serie de 100 casos, se analizaron datos epidemiológicos, clínicos, endocrinológicos, visuales y radiológicos antes y después del tratamiento quirúrgico, y se recogen las complicaciones relacionadas con la cirugía y el seguimiento a largo plazo.

Resultados. El síntoma más frecuente en el momento del diagnóstico fue la afectación del campo visual (62\%), y sólo el $7 \%$ de los adenomas se trataba de un hallazgo casual. El déficit hormonal más frecuente era el hipogonadismo hipogonadótropo (48\%). Tras la cirugía se observó recuperación completa del defecto campimétrico en el 54,8\% de los pacientes, con sólo un $1 \%$ de empeoramiento tras la cirugía, y la incidencia de diabetes insípida fue del $4 \%$. La resección fue superior al $95 \%$ en el $63 \%$ de los casos, a pesar de que el porcentaje de adenomas con invasión del seno cavernoso en grados altos fue elevado (45\%).

Conclusiones. Aunque el síntoma más frecuente de los adenomas hipofisarios no funcionantes es la afectación campimétrica, ésta tiene una excelente respuesta a la cirugía si se realiza dentro del tiempo adecuado. El grado de invasión del seno cavernoso parece el factor más limitante para una resección quirúrgica completa.

Palabras clave. Adenoma no funcionante. Endoscopia endonasal. Hemianopsia. Hipófisis. Knosp. Seno cavernoso.

\section{Introducción}

La cirugía mediante endoscopia endonasal transesfenoidal es el abordaje más utilizado en la actualidad para el tratamiento de los adenomas hipofisarios no funcionantes, tras haber ido ganando terreno durante los últimos años a la misma vía de abordaje, pero realizada con microscopio [1]. Existen numerosas publicaciones en las que se busca correlacionar factores preoperatorios con el grado de resección quirúrgica de los adenomas hipofisarios, pero encontramos una gran variabilidad en las diferentes series publicadas en cuanto al grado de resección, diagnóstico, características morfológicas de cada serie y datos preoperatorios que se recogen en cada uno de ellos [2-5], aunque el factor común a todas las series que lo recogen es la influencia negativa que tiene la invasión del seno cavernoso (habitualmente a través del grado de Knosp).

Muchos de los síntomas que refieren estos pacientes en el momento del diagnóstico son reversibles si el tratamiento se realiza en el momento ade- cuado, por lo que la clave del éxito, como casi siempre, es la indicación correcta. Además de la valoración neurológica y el estudio neurooftalmológico, es imprescindible la colaboración de endocrinología para corregir los déficits hormonales que puedan existir antes y después de la cirugía $[3,6]$.

\section{Pacientes y métodos}

En el Hospital Universitario La Paz de Madrid se han realizado 362 procedimientos de cirugía endoscópica endonasal de base de cráneo. De ellos, en 261 casos, el diagnóstico de la patología que se iba a tratar fue adenoma de hipófisis, de los cuales 172 eran adenomas no funcionantes.

Analizamos una serie de 100 adenomas hipofisarios no funcionantes intervenidos con un abordaje endoscópico endonasal transesfenoidal. Catorce casos se analizaron prospectivamente y 86 retrospectivamente, todos ellos operados por el mismo equipo quirúrgico, siguiendo las mismas indicaciones, y
Servicio de Neurocirugía (C. PérezLópez, A.J. Palpán, A. Zamarrón, A. Isla); Servicio de Otorrinolarin gología (C. Alfonso); Servicio de Endocrinología (C. Álvarez-Escolá, B. Lecumberri); Hospital Universitario La Paz; Madrid. Servicio de Neurología; Hospital Universitario Infanta Sofía; San Sebastián de los Reyes, Madrid, España (M.J. Abenza-Abildúa).

\section{Correspondencia:}

Dr. Carlos Pérez López. Servicio de Neurocirugía. Hospital Universitario La Paz. Paseo de la Castellana, 261. E-28046 Madrid.

E-mail:

cperezhulp@yahoo.es

Aceptado tras revisión externa: 02.06.20.

Cómo citar este artículo:

Pérez-López C, Palpán AJ, AbenzaAbildúa MJ, Zamarrón A, Alfonso C, Álvarez-Escolá C, et al. Adenomas hipofisarios no funcionantes: epidemiología, clínica y evolución posquirúrgica. Rev Neurol 2020; 71: 163-70. doi: 10.33588/rn.7105. 2020273.

(c) 2020 Revista de Neurología 
Figura 1. Imágenes de resonancia magnética con los diferentes grados de Knosp: 0: no invade el seno cavernoso (no sobrepasa la línea tangencial que une la pared medial de la arteria carótida interna supracavernosa con la carótida interna intracavernosa); 1: sobrepasa la tangente medial (definida como la tangente que une los dos bordes mediales de la carótida supra e intracavernosa), pero no sobrepasa la línea tangencial que une los dos centros de la carótida; 2 : se extiende por fuera de la línea intercarotídea, sin sobrepasar la tangente lateral de la carótida intrasupracavernosa; 3: se extiende lateralmente a la línea tangencial lateral que une la porción carotídea supracavernosa con la intracavernosa; 4: la carótida está totalmente englobada por el tumor.

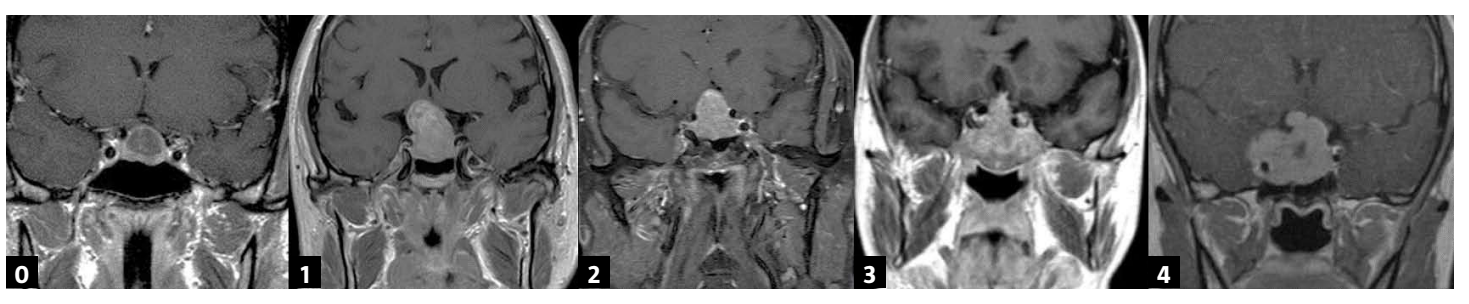

empleando el mismo procedimiento y protocolo en todos ellos.

Se incluyeron pacientes adultos ( $\geq 18$ años) con el diagnóstico de sospecha de adenoma hipofisario no funcionante que cumplían al menos uno de los siguientes criterios:

- Sintomatología neurológica.

- Déficit hormonal.

- Adenomas que contactaban con la vía óptica.

- Adenomas en los que, tras decidirse un tratamiento expectante, se observó un crecimiento tumoral durante el seguimiento.

Además de los datos epidemiológicos y de seguimiento, se estudiaron los antecedentes de cirugía sinusal o selar previa, o de radioterapia hipofisaria. En cuanto a los datos clínicos, se incluyeron los siguientes:

- Clínica preoperatoria.

- Estado hormonal preoperatorio y postoperatorio a los 2 y a los 12 meses. Se analizaron los niveles plasmáticos de factor de crecimiento pseudoinsulínico de tipo 1, cortisol (09:00 h), prolactina, hormona estimulante de la tiroides, tiroxina, hormona estimulante del folículo/hormona luteinizante, estradiol (mujeres) y testosterona (hombres).

- Examen oftalmológico preoperatorio y postoperatorio precoz y a los seis meses, incluyendo una campimetría computarizada SITA 24-2, fondo de ojo, estudio de la motilidad ocular y tomografía de coherencia óptica.

A todos los pacientes se les realizó una resonancia magnética, incluyendo cortes axiales, coronales y sagitales de $2 \mathrm{~mm}$, en máquinas de 1,5 o $3 \mathrm{~T}$. Se compararon los datos obtenidos preoperatoriamen- te y el control posquirúrgico entre los tres y los seis meses. Se valoraron en ambos momentos:

- Volumen de la lesión: medido en mililitros con el software Smartbrush de Brainlab, salvo algunos casos iniciales en los que se hizo con Slicer 3D [7]. Esto permitía medir con precisión el porcentaje resecado (grado de resección), que, a efectos de análisis, se dividió en resección completa (100\%), casi total (> 95\%), subtotal (70-95\%) y parcial $(<70 \%)$. Creemos que este método es el único fiable, ya que medir únicamente la dimensión máxima de la lesión o utilizar el modelo elipsoide $(\mathrm{A} \times \mathrm{B} \times \mathrm{C}) / 2$ puede incurrir en $\mathrm{mu}$ chos errores porque, salvo los adenomas de pequeño tamaño, la mayoría son irregulares, tienen nódulos expansivos y pueden englobar arterias carótidas, con lo cual la estimación del volumen es muy inexacta con este método.

- Dimensión máxima de la lesión en cualquiera de las tres proyecciones.

- Expansión supraselar o invasión de clivus.

- Grado de invasión del seno cavernoso mediante la clasificación de Knosp [8] en los cortes coronales de la resonancia magnética (Fig. 1).

- Presencia y porcentaje de hemorragia o componente quístico intratumoral del volumen total del adenoma.

En el seguimiento de complicaciones postoperatorias inmediatas y tardías se recogieron, además de los controles de imagen, los estudios hormonales y neurooftalmológicos mencionados con anterioridad. También se incluyó, dentro del seguimiento a largo plazo, la posible necesidad de una reintervención quirúrgica o la aplicación de radiocirugía.

Se realizó el análisis estadístico con Microsoft Excel 2019 y el programa SPSS v. 25.0. 
Tabla l. Situación hormonal (preoperatoria y a los 2 y 12 meses tras la cirugía), evolución hormonal entre el preoperatorio y a los 12 meses (aparición de nuevas alteraciones hormonales/curación de alteraciones preoperatorias), y neurooftalmológica (antes de la cirugía y 1 y 6 meses después de la intervención).

\begin{tabular}{|c|c|c|c|c|c|c|}
\hline & & Preop. & 2 meses & 12 meses & Curación & Nuevo déficit \\
\hline \multirow{7}{*}{$\begin{array}{l}\text { Déficit } \\
\text { hormonal (\%) }\end{array}$} & Hipogonadismo hipogonadótropo & 48 & 45 & 46 & $7 / 48(14,6 \%)$ & $5 / 52(9,6 \%)$ \\
\hline & Hipotiroidismo secundario & 43 & 46 & 45 & $2 / 43(4,7 \%)$ & $4 / 57(7 \%)$ \\
\hline & Insuficiencia suprarrenal secundaria & 28 & 55 & 37 & $4 / 28(14,3 \%)$ & $13 / 72(18 \%)$ \\
\hline & Déficit de hormona del crecimiento & 32 & 33 & 32 & $2 / 32(6,3 \%)$ & $2 / 68(2,9 \%)$ \\
\hline & Hiperprolactinemia & 26 & 0 & 0 & $26 / 26(100 \%)$ & $0 / 0$ \\
\hline & Diabetes insípida & 0 & 11 & 4 & 0 & $4 / 100(4 \%)$ \\
\hline & & Preop. & 1 mes & 6 meses & & \\
\hline \multirow{9}{*}{$\begin{array}{l}\text { Variable neuro- } \\
\text { oftalmológica (\%) }\end{array}$} & Defecto campimétrico & 62 & 41 & 28 & & \\
\hline & Hemianopsia & 43 & 5 & 4 & & \\
\hline & Cuadrantanopsia & 19 & 36 & 24 & & \\
\hline & Afectación de los pares craneales & 9 & 5 & 1 & & \\
\hline & III & 8 & 4 & 0 & & \\
\hline & IV & 1 & 0 & 0 & & \\
\hline & V1 & 0 & 0 & 0 & & \\
\hline & V2 & 0 & 0 & 0 & & \\
\hline & VI & 5 & 3 & 1 & & \\
\hline
\end{tabular}

\section{Resultados}

\section{Epidemiología}

De los 100 pacientes incluidos en el estudio, 45 eran mujeres y 55 eran varones, con una edad media de 54,7 años (rango: 18-84 años) y un seguimiento medio de 72,5 meses (rango: 6-164 meses). En cuanto a la anatomía patológica, siete de los 100 adenomas resultaron ser atípicos.

En cuanto a los antecedentes personales, el 20\% había recibido previamente cirugía hipofisaria; el $6 \%$, cirugía sinusal; y el $3 \%$ había sido radiado previamente. El síntoma preoperatorio más frecuente fue el déficit visual (62\%), seguido de cefalea (42\%). El siguiente grupo de síntomas más frecuente es el relacionado con el déficit hormonal (amenorreagalactorrea, 12\%; disminución de la libido, 11\%; y astenia, 9\%, son los más frecuentes). En el 7\% de los pacientes, el diagnóstico de la lesión se hizo a raíz de una apoplejía, y el $2 \%$ comenzó con un bajo nivel de conciencia. El grupo de pacientes en los que el adenoma fue un hallazgo casual (incidentaloma) supuso el $7 \%$ del total.

\section{Endocrinología}

La tabla I recoge la información relevante respecto a la situación hormonal. Además de estudiar los déficits hormonales de cada caso, se ha incluido su evolución en cuanto al número de casos de aparición de nuevos déficits y de correcciones de alteraciones previas a la cirugía. El déficit preoperatorio más frecuente fue el hipogonadismo hipogonadótropo (48\%), y el déficit que con mayor frecuencia se produjo con la cirugía fue la insuficiencia suprarrenal secundaria (13 casos). La corrección se produjo en los 26 casos de hiperprolactinemia por compresión del tallo.

\section{Neurooftalmología}

La afectación del campo visual fue la hemianopsia (uni o binocular), detectada en un $43 \%$ del total de casos. A los seis meses, 34 pacientes (54,8\%) habían normalizado completamente el campo visual, y sólo había cuatro hemianopsias. Sólo un paciente empeoró tras la cirugía. De los nueve pacientes que tenían afectación de los pares oculomotores, todos, 
Figura 2. Evolución posquirúrgica respecto al grado de Knosp.

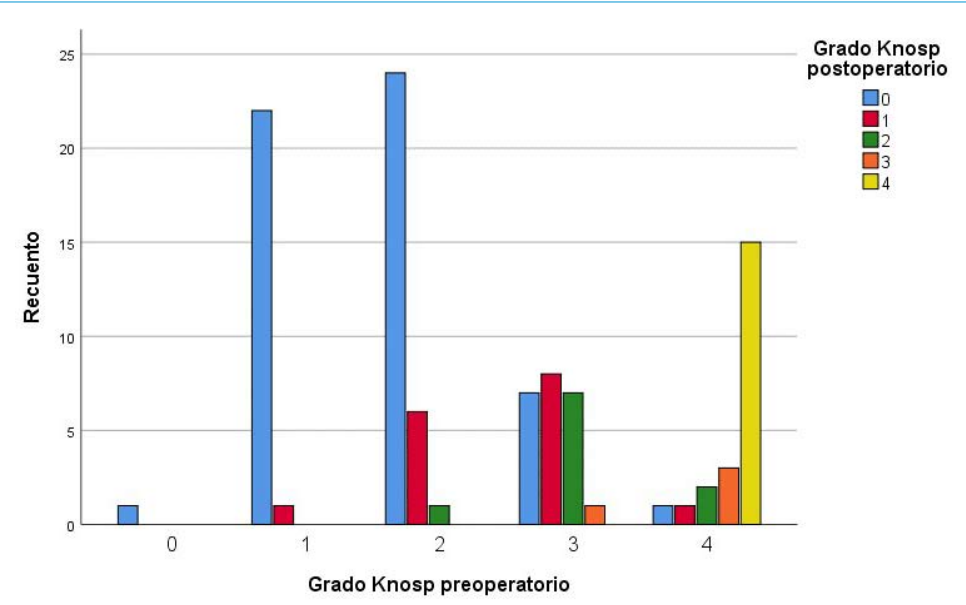

Tabla II. Resumen de los datos radiológicos más relevantes: volúmenes tumorales pre y posquirúrgicos, grado de resección y dimensión tumoral máxima.

\begin{tabular}{|c|c|c|c|c|c|}
\hline & & Mínimo & Máximo & Mediana & $\%(n=100)$ \\
\hline \multicolumn{2}{|c|}{ Volumen prequirúrgico $(\mathrm{mL})$} & 0,5 & 58 & 6,1 & \\
\hline \multicolumn{2}{|c|}{ Volumen posquirúrgico $(\mathrm{mL})$} & 0 & 6,9 & 0,1 & \\
\hline \multicolumn{2}{|c|}{ Grado de resección (\%) } & 46,05 & 100 & 98,88 & \\
\hline \multicolumn{2}{|c|}{ Dimensión máxima (mm) } & 7 & 67 & 27 & \\
\hline \multirow{4}{*}{$\begin{array}{l}\text { Grado de } \\
\text { resección }\end{array}$} & Completa (100\%) & & & & $49 \%$ \\
\hline & Casi completa (95-99,9\%) & & & & $14 \%$ \\
\hline & Subtotal (70-94,99\%) & & & & $24 \%$ \\
\hline & Parcial (< 70\%) & & & & $13 \%$ \\
\hline
\end{tabular}

excepto uno, habían recuperado el déficit a los seis meses. Los detalles se muestran en la tabla I.

\section{Pruebas de imagen}

Encontramos expansión supraselar del adenoma en el $85 \%$ de los pacientes e invasión del clivus en el $32 \%$. Respecto al contenido del adenoma, el $28 \%$ tenía un contenido hemorrágico menor del 50\%; el $12 \%$, mayor del 50\%; y el $60 \%$ carecía de él. Encontramos un componente quístico menor del $50 \%$ en el $22 \%$ de los casos; mayor del $50 \%$, en el $8 \%$; y el $70 \%$ de los adenomas estudiados no tenía ningún tipo de componente quístico.
Los datos de volúmenes tumorales pre y posquirúrgicos, el grado de resección y la dimensión tumoral máxima están recogidos en la tabla II.

La figura 2 muestra la correlación entre los grados de Knosp pre y postoperatorio, y se observa que las reducciones en el grado de Knosp son mayores en los grados más bajos: de los 23 casos con Knosp 1 preoperatorio, casi todos ellos (22 casos) se convertían en Knosp 0; en cambio, de los 22 casos de Knosp 4, 15 seguían en el grado 4 tras la cirugía.

\section{Complicaciones posquirúrgicas}

No se produjeron lesiones vasculares ni mortalidad.

La complicación más grave fue una meningitis resuelta sin secuelas, y la más frecuente en el postoperatorio inmediato fue la complicación endocrinológica (el 16\% de los casos, con 10 diabetes insípidas, cuatro síndromes de secreción inadecuada de hormona antidiurética y dos respuestas trifásicas), aunque sólo cuatro de esos pacientes sufrieron una diabetes insípida permanente.

Sólo hubo un caso de dudosa fístula de líquido cefalorraquídeo, que se resolvió espontáneamente en 24 horas. En relación con las fístulas, se observó salida de líquido cefalorraquídeo intraoperatoria en el 29\% de los casos, y para la reconstrucción se empleó una combinación de los siguientes elementos: grasa (35\%), sustituto dural (43\%), sellante (94\%), flap pediculado nasoseptal (37\%) y flap libre de mucosa del cornete medio (50\%).

En el 7\% de los casos, los pacientes referían cefalea intensa en el postoperatorio inmediato, que casi siempre coincidió con el hallazgo de un hematoma en el lecho quirúrgico (7\%), aunque ninguno requirió tratamiento quirúrgico porque no repercutía sobre la función visual.

Una paciente presentó empeoramiento visual de un ojo tras la cirugía, probablemente por causas isquémicas. También se observó epistaxis en el 5\% de los casos y sinusitis en el $4 \%$.

\section{Seguimiento}

Durante el seguimiento de estos pacientes, 12 fueron reintervenidos en un rango temporal entre cuatro y 102 meses. Diecisiete pacientes de la serie recibieron radiocirugía, en 16 de los cuales se planteó como tratamiento complementario a la cirugía, y en uno como tratamiento al crecimiento de un resto tumoral en el que se consideró que la resección quirúrgica iba a ser escasa y poco rentable. Sólo se reintervinieron dos de los siete adenomas atípicos que había. 


\section{Discusión}

\section{Epidemiología}

Los adenomas hipofisarios no funcionantes son tumores benignos que surgen de la adenohipófisis y no se acompañan de evidencia clínica o bioquímica de exceso hormonal, a excepción de hiperprolactinemia leve en algunos casos. Representan el 14-54\% de los adenomas hipofisarios [9-14]. Estudios de población del Reino Unido, Bélgica, Finlandia, Suecia, Malta, Islandia, Canadá y Argentina han estimado que la prevalencia de los adenomas hipofisarios no funcionantes clínicamente relevantes es de 7-41,3 casos por cada 100.000 habitantes (Tabla III) [9-20]. En la mayoría de los estudios epidemiológicos, es el segundo tipo de adenoma más frecuente después de los prolactinomas, aunque cuando hablamos solamente de macroadenomas, es el grupo más habitual. Los datos son discordantes en cuanto al sexo más frecuente, y tienen una tasa de incidencia estandarizada de 0,65-2,34/100.000, cuya incidencia máxima se encuentra entre la cuarta y la octava décadas de la vida [9].

\section{Clínica}

Los síntomas en el momento del diagnóstico son similares a los referidos en otras series, salvo que nosotros observamos un porcentaje de afectación visual (62\%) superior a muchas de ellas, y sólo un 7\% de cirugía en los incidentalomas (inferior a lo habitual) [21]. Esto se debe a que no se indicaba cirugía en los adenomas hipofisarios no funcionantes salvo que produjesen síntomas o déficits hormonales, contactaran con la vía óptica o hubieran crecido durante el seguimiento, y seguimos creyendo que es el tratamiento correcto, ya que el crecimiento de muchos adenomas es nulo o mínimo y, si el seguimiento es adecuado, se puede hacer la cirugía antes de que pueda aparecer una afectación visual, ya que los cambios en la resonancia magnética suelen preceder a los cambios clínicos visuales [22-24].

\section{Endocrinología}

En cuanto a las alteraciones hormonales detectadas preoperatoriamente, todas ellas se encuentran dentro de las incidencias publicadas, aunque en el límite bajo $[6,25]$. Llama la atención nuestra baja incidencia de déficit de la hormona del crecimiento (32\%) respecto a lo publicado en la bibliografía [26]. Creemos que dicha discrepancia se debe a que el diagnóstico de déficit de la hormona del crecimien-
Tabla III. Prevalencia y demografía de los adenomas hipofisarios no funcionantes sintomáticos.

\begin{tabular}{lccccc}
\hline & País & Período & $\begin{array}{c}\text { Prevalencia } \\
(\times 100.000 \text { hab. })\end{array}$ & $\begin{array}{c}\text { Mujeres/ } \\
\text { hombres }\end{array}$ & $\begin{array}{c}\text { Edad } \\
\text { (años) }\end{array}$ \\
\hline Daly et al [11] & Bélgica & 2005 & 13,8 & $3 / 7$ & 61,5 \\
\hline Fernández et al [10] & Reino Unido & 2006 & 22,1 & $6 / 12$ & 51,5 \\
\hline Raappana et al [16] & Finlandia & $1992-2007$ & $22,2-26,5$ & $5 / 4$ & 60 \\
\hline Gruppetta et al [12] & Malta & $2000-2011$ & 25,8 & $119 / 27$ & 47 \\
\hline Tjörnstrand et al [13] & Suecia & $2001-2011$ & 22 & $135 / 185$ & 51,5 \\
\hline Al-Dahmani et al [18] & Canadá & $2005-2013$ & 41,3 & $385 / 215$ & 52,1 \\
\hline Agustsson et al [17] & Islandia & $1955-2012$ & 41,32 & $99 / 104$ & 57 \\
\hline Davis et al [20] & Argentina & $2003-2014$ & 21,48 & $19 / 10$ & 68,7 \\
\hline
\end{tabular}

to no es igual en todas las series, al estar influida su interpretación por la edad del paciente y los estándares habituales de cada laboratorio. De los déficits hormonales producidos en la cirugía, el más frecuente fue la insuficiencia suprarrenal, con un $9 \%$ a los 12 meses, aunque a los dos meses este incremento había sido casi hasta el doble, lo que pone de manifiesto el hecho de que, en muchos casos, el hipocortisolismo postoperatorio es transitorio. Además, a todos los pacientes se les administraba hidroaltesona de maanera profiláctica y esto probablemente hizo que su retirada se demorara más de lo necesario en algunos casos. Dicha transitoriedad del déficit también hay que tenerla en cuenta para la diabetes insípida: un $11 \%$ de casos nuevos a los dos meses y un $4 \%$ a los seis meses.

\section{Neurooftalmología}

La evolución visual fue muy satisfactoria en casi todos los pacientes, y destacó sólo un caso de empeoramiento con la cirugía. La recuperación de otros pares craneales se había logrado casi siempre (ocho de nueve) a los seis meses. El empeoramiento se produjo en una reintervención de una paciente con miopía magna y una maculopatía; creemos que se debió a una isquemia que afectó a la visión de un ojo. Nuestros resultados se encuentran en el límite alto de mejoría respecto a la bibliografía, lo cual creemos que se debe a un control oftalmológico estricto y al valor pronóstico de la tomografía de coherencia óptica, que marca la necesidad de una cirugía precoz en casos reversibles [27]. 


\section{Pruebas de imagen}

Se alcanzó la resección completa solamente en el $49 \%$ de los casos, cifra que se encuentra en el límite bajo de la horquilla en cuanto a las publicaciones existentes [5,21,28-30]. Si ampliamos el grado de resección a los casos con exéresis superior al 95\%, el porcentaje de resecciones ascendería al 63\%. Hay varios factores que explican esta tasa de resección inferior a muchas de las publicadas:

- El 45\% de los adenomas eran de grado de Knosp 3 o 4 . En la mayoría de las series quirúrgicas publicadas no nos dan información de este dato, que es el más limitante para realizar una resección completa, y en los que tenemos el dato, como en el estudio TRANSSPHER [21], donde se logró una resección completa en el 81,5\% de casos, sólo el 18,4\% de los adenomas eran de grado de Knosp 3 o 4.

- Consideramos la existencia de un resto tumoral cuando al menos en un corte de la resonancia magnética (cualquier secuencia y proyección) se veía una imagen sospechosa. Por ejemplo, en el estudio mencionado en el punto anterior, se consideraba que había tumor residual sólo si se veía en dos cortes consecutivos y se correlacionaba con la misma imagen en otro plano.

- En nuestra serie incluimos los 100 primeros casos de adenoma hipofisario no funcionante operados por un mismo cirujano, con lo cual abarcamos gran parte de nuestra curva de aprendizaje.

Por tanto, creemos que la diferencia viene dada por la mayor frecuencia de invasión del seno cavernoso en los grados altos de Knosp, porque nuestra interpretación de las imágenes ha sido más 'penalizadora' y, por supuesto, porque nuestra experiencia no es la misma que la de los neurocirujanos del artículo con el que comparamos nuestra serie.

\section{Complicaciones}

Sólo se observó un caso de dudosa fístula de líquido cefalorraquídeo, que se resolvió espontáneamente en 24 horas, a pesar de que se observó salida intraoperatoria de líquido cefalorraquídeo en el $29 \%$ de casos. La clave para minimizar el riesgo de fístula es adaptar nuestra reconstrucción a cada caso. En general, si creemos que va a haber una salida importante de líquido cefalorraquídeo, se planea un flap pediculado nasoseptal, y en el lecho quirúrgico colocamos grasa (a veces con duramadre artificial) que cubrimos con el flap obtenido. Si no prevemos una salida importante de líquido cefalorraquídeo, no se diseña un flap pediculado y se coloca un flap libre de mucosa de cornete medio. Si ha habido salida de líquido, colocamos también grasa y a veces duramadre sintética por debajo de la mucosa libre. En todos los casos, tras un tipo u otro de flap, aplicamos un sellante pulverizado. Creemos importante no hacer el flap pediculado, salvo que estemos seguros de que será muy necesario, ya que vemos que con el flap libre es suficiente en la mayoría de los casos, y así evitamos dejar mucha área del tabique desnudo de mucosa, y disminuimos las costras y el riesgo de perforación de tabique.

Todos los casos que sufrieron epistaxis postoperatoria habían estado en tratamiento con antiagregantes o anticoagulantes, por lo que hay que prestar especial atención a la hemostasia nasal en estos pacientes. Hemos encontrado una alta incidencia (7\%) de hematoma en el lecho quirúrgico, aunque ninguno de ellos con afectación visual, por lo que no fueron reintervenidos. Creemos que la razón más importante de este hallazgo es por la entrada de sangre a través de un desgarro intraoperatorio del diafragma selar y, por ese motivo, el hematoma no está en tensión y no produce compresión de la vía óptica, sino cefalea irritativa. De hecho, en todos los casos, excepto en uno, se había observado salida de líquido cefalorraquídeo intraoperatoriamente. Por este motivo, al resecar un adenoma de hipófisis, la última parte que debemos resecar es la más superior, para evitar que entre sangre ante una eventual fístula de líquido cefalorraquídeo intraoperatoria, y para que, en los casos en los que el diafragma esté muy estirado y descienda de forma importante en la silla, no nos impida realizar la extirpación del adenoma con comodidad.

La tasa global de complicaciones es baja y comparable a las mejores series de la bibliografía $[21,31$, 32], y destaca la ausencia de mortalidad y de complicaciones graves.

\section{Seguimiento}

Doce pacientes fueron reintervenidos durante el seguimiento, dos de ellos al principio de la serie, por resección insuficiente, probablemente ante la falta de experiencia quirúrgica, y 10 a lo largo del tiempo. No es una proporción muy alta si tenemos en cuenta que el seguimiento medio fue de 72,5 meses. No se observó relación entre el recrecimiento del resto y el hecho de que se tratase de un adenoma atípico, y sólo se reoperó un adenoma que había recibido radiocirugía previamente (era un adenoma típico). Hay que tener en cuenta que, tras la primera cirugía, se radiaron 17 pacientes, todos de forma 
inmediata, salvo uno en el que se observó crecimiento de un pequeño resto que englobaba la arteria carótida interna y en el que se consideraba que la cirugía iba ser poco rentable en términos de resección. Recibieron radiocirugía los adenomas atípicos y los que tenían resto tumoral significativo en el seno cavernoso, ya que consideramos que una nueva cirugía tampoco volvería a lograr una resección total o casi total.

\section{Limitaciones del estudio}

La principal limitación es el número de pacientes, si lo comparamos con algunas de las series publicadas, lo que hace que algunas variables hayan sido desestimadas por un número bajo de casos y otras ni se hayan podido analizar. Otro detalle a tener en cuenta es el hecho de que abarcar parte del período inicial de la curva de aprendizaje puede ser un obstáculo, aunque creemos que, lejos de ser un problema, permite la aplicabilidad en más centros y puede por tanto ser un valor añadido, dependiendo de la experiencia quirúrgica del lector.

La mayoría de los casos de la serie se ha recogido de forma retrospectiva, y tanto la recogida de datos como la valoración de las pruebas de imagen la han realizado los autores del trabajo.

En conclusión, un alto grado de Knosp preoperatorio es el factor más limitante para el grado de resección quirúrgica. Los síntomas visuales son reversibles si el paciente recibe tratamiento quirúrgico precoz, aunque los déficits hormonales se corrigen en un porcentaje muy leve. Las complicaciones de la endoscopia endonasal transesfenoidal son pocas y poco graves en la mayoría de los casos, y destaca la baja incidencia de fístulas de líquido cefalorraquídeo.

\section{Bibliografía}

1. Fernández-Miranda JC, Prevedello DM, Gardner P, Carrau R, Snyderman CH, Kassam AB. Endonasal endoscopic pituitary surgery: is it a matter of fashion? Acta Neurochir (Wien) 2010; 152: $1281-2$

2. Murad MH, Fernández-Balsells MM, Barwise A, GallegosOrozco JF, Paul A, Lane MA, et al. Outcomes of surgical treatment for nonfunctioning pituitary adenomas: a systematic review and meta-analysis. Clin Endocrinol (Oxf) 2010; 73: 777-91.

3. Ammirati M, Wei L, Ciric I. Short-term outcome of endoscopic versus microscopic pituitary adenoma surgery: a systematic review and meta-analysis. J Neurol Neurosurg Psychiatry 2013; 84: 843-9.

4. Mooney MA, Sarris CE, Zhou JJ, Barkhoudarian G, Chicoine MR, Fernández-Miranda JC, et al. Proposal and validation of a simple grading scale (TRANSSPHER grade) for predicting gross total resection of nonfunctioning pituitary macroadenomas after transsphenoidal surgery. Oper Neurosurg 2019; 17: 460-9.
5. Paluzzi A, Fernández-Miranda JC, Tonya Stefko S, Challinor S, Snyderman CH, Gardner PA. Endoscopic endonasal approach for pituitary adenomas: a series of 555 patients. Pituitary 2014; 17: 307-19.

6. Wichers-Rother M, Hoven S, Kristof RA, Bliesener N, Stoffel-Wagner B. Non-functioning pituitary adenomas: endocrinological and clinical outcome after transsphenoidal and transcranial surgery. Exp Clin Endocrinol Diabetes 2004; 112: 323-7.

7. Egger J, Kapur T, Nimsky C, Kikinis R. Pituitary adenoma volumetry with 3D slicer. PLoS One 2012; 7: e51788.

8. Knosp E, Steiner E, Kitz K, Matula C. Pituitary adenomas with invasion of the cavernous sinus space: a magnetic resonance imaging classification compared with surgical findings. Neurosurgery 1993; 33: 610-7.

9. Ntali G, Wass JA. Epidemiology, clinical presentation and diagnosis of non-functioning pituitary adenomas. Pituitary 2018; 21: 111-8.

10. Fernández A, Karavitaki N, Wass J. Prevalence of pituitary adenomas: a community-based, cross-sectional study in Banbury (Oxfordshire, UK). Clin Endocrinol (Oxf) 2010; 72: 377-82.

11. Daly AF, Rixhon M, Adam C, Dempegioti A, Tichomirowa MA, Beckers A. High prevalence of pituitary adenomas: a crosssectional study in the province of Liège, Belgium. J Clin Endocrinol Metab 2006; 91: 4769-75.

12. Gruppetta M, Mercieca C, Vassallo J. Prevalence and incidence of pituitary adenomas: a population based study in Malta. Pituitary 2013; 16: 545-53.

13. Tjörnstrand A, Gunnarsson K, Evert, Holmberg E, Ragnarsson O, Rosén $\mathrm{T}$, et al. The incidence rate of pituitary adenomas in western Sweden for the period 2001-2011. Eur J Endocrinol 2014; 171: 519-26.

14. Fainstein Day P, Loto MG, Glerean M, Picasso MFR, Lovazzano S, Giunta DH. Incidence and prevalence of clinically relevant pituitary adenomas: retrospective cohort study in a health management organization in Buenos Aires, Argentina. Arch Endocrinol Metab 2016; 60: 554-61.

15. Fontana E, Gaillard R. Epidemiology of pituitary adenoma: results of the first Swiss study. Rev Med Suisse 2009; 5: 2172-4.

16. Raappana A, Koivukangas J, Ebeling T, Pirilä T. Incidence of pituitary adenomas in Northern Finland in 1992-2007. J Clin Endocrinol Metab 2010; 95: 4268-75.

17. Agustsson TT, Baldvinsdottir T, Jonasson JG, Olafsdottir E, Steinthorsdottir V, Sigurdsson G, et al. The epidemiology of pituitary adenomas in Iceland, 1955-2012: a nationwide population-based study. Eur J Endocrinol 2015; 173: 655-64.

18. Al-Dahmani K, Mohammad S, Imran F, Theriault C, Doucette S, Zwicker D, et al. Sellar masses: an epidemiological study. Can J Neurol Sci 2016; 43: 291-7.

19. Davis JRE, Farrell WE, Clayton RN. Pituitary tumours. Reproduction 2001; 121: 363-71.

20. Faglia G. Epidemiology and pathogenesis of pituitary adenomas. Acta Endocrinol (Copenh) 1993; 129 (Suppl 1): S1-5.

21. Little AS, Kelly DF, White WL, Gardner PA, FernándezMiranda JC, Chicoine MR, et al. Results of a prospective multicenter controlled study comparing surgical outcomes of microscopic versus fully endoscopic transsphenoidal surgery for nonfunctioning pituitary adenomas: the Transsphenoidal Extent of Resection (TRANSSPHER) Study. J Neurosurg 2019; 22: 1-11.

22. Sam AH, Shah S, Saleh K, Joshi J, Roncaroli F, Robinson S, et al. Clinical outcomes in patients with nonfunctioning pituitary adenomas managed conservatively. Clin Endocrinol (Oxf) 2015; 83: 861-5.

23. Sivakumar W, Chamoun R, Nguyen V, Couldwell WT. Incidental pituitary adenomas. Neurosurg Focus 2011; 31: E18.

24. Scangas GA, Laws ER. Pituitary incidentalomas. Pituitary 2014; 17: 486-91.

25. Cury ML, Fernandes JC, Machado HR, Elias LL, Moreira AC, Castro MD. Non-functioning pituitary adenomas: clinical feature, laboratorial and imaging assessment, therapeutic management and outcome. Arq Bras Endocrinol Metabol 2009; 53: 31-39. 
26. Fleseriu M, Bodach ME, Tumialan LM, Bonert V, Oyesiku NM, Patil CG, et al. Guidelines: congress of neurological surgeons systematic review and evidence-based guideline for pretreatment endocrine evaluation of patients with nonfunctioning pituitary adenomas. Neurosurgery 2016; 79: E527-9.

27. Jacob M, Raverot G, Jouanneau E, Borson-Chazot F, Perrin G, Rabilloud M, et al. Predicting visual outcome after treatment of pituitary adenomas with optical coherence tomography. Am J Ophthalmol 2009; 147: 64-70.

28. Sanmillán JL, Torres-Díaz A, Sánchez-Fernández JJ, Lau R, Ciller C, Puyalto P, et al. Radiologic predictors for extent of resection in pituitary adenoma surgery. A single-center study. World Neurosurg 2017; 108: 436-46.

29. Cappabianca P, Cavallo LM, Divitiis O, Solari D, Esposito F,
Colao A. Endoscopic pituitary surgery. Pituitary 2008; 11: 385-90.

30. Almutairi RD, Muskens IS, Cote DJ, Dijkman MD, Kavouridis VK, Crocker E, et al. Gross total resection of pituitary adenomas after endoscopic vs. microscopic transsphenoidal surgery: a meta-analysis. Acta Neurochir (Wien) 2018; 160: 1005-21.

31. Cappabianca P, Cavallo LM, Colao A, Del Basso De Caro M, Esposito F, et al. Endoscopic endonasal transsphenoidal approach: outcome analysis of 100 consecutive procedures. Minim Invasive Neurosurg 2002; 45: 193-200.

32. Ciric I, Ragin A, Baumgartner C, Pierce D. Complications of transsphenoidal surgery: results of a national survey, review of the literature, and personal experience. Neurosurgery 1997 40: 225-37.

\section{Non-functioning pituitary adenomas: epidemiology, clinical and postoperative outcome}

Introduction. Non-functioning pituitary adenomas are the most frequent tumor group in the sellar region. They are usually benign neoplasms diagnosed after visual or hormonal symptoms, although it is not uncommon to detect them as a casual finding.

Aim. To analyze the clinical aspects found in this disease and its response after surgical treatment.

Patients and methods. In a series of 100 cases, epidemiological, clinical, endocrinological, visual and radiological data were analyzed before and after surgical treatment, as well as the complications related to surgery and long-term follow-up.

Results. The most frequent symptom at the time of diagnosis was visual field involvement (62\%), and only the $7 \%$ of adenomas were a casual finding. The most common hormonal deficit was hypogonadotropic hypogonadism (48\%). After surgery, complete recovery of the visual field defect was observed in $54.8 \%$ of the patients, only $1 \%$ worsening after surgery, and the incidence of diabetes insipidus was $4 \%$. The resection was superior to $95 \%$ in $63 \%$ of cases, although the percentage of adenomas with invasion of the cavernous sinus in Knosp grades 3 and 4 it was high (45\%).

Conclusions. Although the most frequent symptom of non-functioning pituitary adenomas is campimetric involvement, it has an excellent response to surgery if it is performed within the appropriate time. The grade of invasion of the cavernous sinus is the most limiting factor for a complete surgical resection.

Key words. Cavernous sinus. Endonasal endoscopy. Hemianopia. Knosp. Non-functioning adenoma. Pituitary. 